\title{
MicroRNA 基因簇的多样性与进化
}

\author{
张雁峰，张锐，宿兵" \\ 中国科学院昆明动物研究所, 遗传资源与进化国家重点实验室, 昆明 650223 \\ * 联系人, E-mail: sub@mail.kiz.ac.cn \\ 收稿日期: 2008-09-27; 接受日期: 2008-11-28 \\ 国家自然科学基金重点项目(批准号: 306300130)资助
}

\begin{abstract}
摘要 microRNA(miRNA)基因簇是一类 miRNA 基因在染色体上成簇排列形成的基因群. 在动植物基因组中, 已发现存在大量簇生排列的 miRNA 基因. 然而这种簇生排列方式所具 有的功能意义尚不完全清楚. miRNA 基因簇常位于一个多顺反子内, 通过共表达在胚胎发 育、细胞周期和细胞分化等诸多方面发挥协同调控的功能. 基于近些年来的研究进展, 本文 综述了 miRNA 基因簇在动物染色体上分布的多样性, 表达与功能的协同性, 以及重复和转 座介导下 miRNA 基因簇产生与消亡的适应性进化过程.

关键词 miRNA 基因簇 基因重复 适应性进化 转座
\end{abstract}

microRNAs(miRNAs) 是一类长度为 $20 \sim 25 \mathrm{nt}$ 的 内源性小RNA分子, 在转录后水平负调控基因的表 达和翻译 ${ }^{[1]}$. 在动物细胞内, miRNA基因由RNA聚合 酶 II [2] 或者RNA聚合酶 III [3] 转录产生初级miRNA转 录物(primary microRNAs) ${ }^{[4]}$, 经Drosha(RNase III蛋白 家族一成员) 和DGCR 8 形成的复合体 ${ }^{[5]}$ 识别和切割, 形成具有发夹结构的长度约 70 90 nt 的前体 miRNA(precursor microRNA). 在Exportin 5 的介导 下 ${ }^{[6]}$, 前体 miRNA从细胞核内被转运出核到细胞质 中, 由另一个位于细胞质内的 RNase III酶—Dicer 识 别发夹结构和切割后形成双链成熟的miRNA. 其中 一条链被快速降解(个别miRNA除外, 比如miR-151 的两条链都发挥着功能), 剩余的一条链在一种称为 RNA诱导沉默复合体(RNA-induced silencing complex, RISC)的介导下按照不完全碱基互补配对的方式与 mRNA的 3'UTR结合, 从而抑制mRNA的翻译, 或降 低mRNA的稳定性，抑或降解mRNA. 这种抑制或降 解的方式主要取决于两者之间互补配对的程度 ${ }^{[4]}$.
有研究显示 ${ }^{[7 ~ 10],}$ miRNA的“种子区”(seed region)(第 2 8 位碱基序列)与 $3^{\prime} U T R$ 的互补配对决定了 miRNA 的抑制程度.

大量研究表明, miRNA在发育时序性调控 ${ }^{[11,12]}$ 、 细胞分化 ${ }^{[13,14]}$ 、抗病毒免疫应答 ${ }^{[15]}$ 、应激反应调节 ${ }^{[16]}$ 等诸多方面起着至关重要的作用. 这种分子开关调控 功能的异常将导致诸如癌症 $[17,18]$ 、神经退行性疾病 ${ }^{[19]}$ 、 心脏病 ${ }^{[200}$ 等疾病的产生.

全基因组水平非编码 DNA 序列的研究发现, miRNA 基因在染色体上的分布是非随机的, 许多 miRNA基因紧密相邻, 排列成簇 $[21,22]$. 关于簇生排列 的miRNA有哪些特殊功能及它们的起源与进化过程 至今仍不是很清楚. 鉴于此, 本文结合最近几年关于 miRNA基因簇的研究进展，阐述了 miRNA基因簇在 动植物基因组中分布的多样性，表达与功能调控作 用的协同性，以及簇生排列的进化模式，并通过具体 的实证性例子分析了 miRNA基因簇产生与消亡 的适应性进化, 对于调控基因表达网络和物种形成 
过程的重要意义.

\section{1 miRNA 基因簇的多样性}

基因簇(gene clusters)是指在染色体上彼此紧密 相邻的两个或者多个基因构成的基因群 ${ }^{[23]}$. 成簇排 列的基因之间可以具有同源性关系，也可不具有任 何同源性. 最近，Lai等人 ${ }^{[22]}$ 已经发现miRNA基因在 黑腹果蝇(Drosophila melanogaster)染色体上的排布 也具有成簇的特征. 这种 miRNA基因成簇排列的方 式提示了 miRNA可能具有复杂的调控机制.

先前研究发现，果蝇基因组中约有 $50 \%$ 的 miRNA基因是成簇排列的，而人类基因组中的 miRNA基因可能只有很少一部分成簇排列 ${ }^{[4]}$. 然而, 这种认识随着在人基因组中越来越多的miRNA被发 现而改变. 设 $3000 \mathrm{nt}$ 为 miRNA基因之间成簇排列的 最大距离(maximum inter-miRNA distance, MID), Altuvia等人 ${ }^{[24]}$ 系统分析了来自 Sanger microRNA数据 库(release 4.0)的 207 个人类miRNAs, 发现了 31 个 miRNA基因簇，共包括 76 个 miRNAs，占到分析总数 的 37\%左右. Megraw等人 ${ }^{[25]}$ 对人 (Homo sapiens) 小鼠 (Mus musculus)、大鼠(Rattus norvegicus)和鸡(Gallus gallus ) 4 个物种基因组中的miRNA基因簇进行了分 析，当定义MID为 $1 \mathrm{~kb}$ 时，超过 30\%的miRNA基因是 呈簇集式分布的. 当MID的限定增至 $50 \mathrm{~kb}$ 时，超过 $1 / 2$ 的miRNA基因在染色体内是以成簇方式排列的. 总体来说，考虑到miRNA基因的定义，成簇分布的 miRNA个数显著高于随机分布的个数. 这暗示了成簇 分布的 miRNA可能共享一些顺式调控元件. $\mathrm{smiRNAdb}$ 数据库 (http://www.mirz.unibas.ch/smiRNAdb/cgi/smiRNAdb?page=home) 限定了 构成 miRNA 基因簇的 miRNA 基因之间的最大距离 为 $50 \mathrm{~kb}$. 根据这一限定, 本文列出了人、小鼠和大鼠 基因组中所有已知的 miRNA 基因簇的数目(图 1).

此外, Tang等人 ${ }^{[26]}$ 通过生物信息学预测方法分析 了miRNA基因在非洲爪蟾(Xenopus laveis)基因组中 的分布情况, 结果发现 miRNA主要位于其基因组的 内含子区域，同时有近 50\%的miRNA基因在染色体 上成簇排列. 更有趣的是，部分 miRNA基因簇尚有不 止 1 份楛贝，形成了重复的miRNA基因簇(repeated clusters), 如在人类中素有癌基因之称的
Mir-17/Mir-92 基因簇 $[17,27]$, 在非洲爪蟾中形成了 miRNA基因簇的重复.

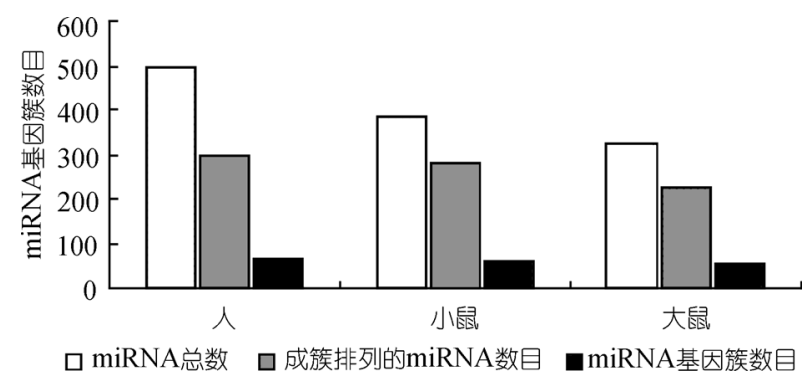

图 1 哺乳动物基因组中 miRNA 基因簇的数目 (http://www.mirz.unibas.ch/smiRNAdb/PreClust.html)

以上研究表明，miRNA基因簇在后生动物基因 组中都有很大比例的分布, 呈现出分布多样性. 当 miRNA基因簇各个成员之间存在同源的关系时，这 样的 miRNA 基因簇就构成了 miRNA 基因家族 (miRNA gene family), 从而进一步扩充了 miRNA基 因簇分布的多样性. 如在秀丽线虫(Caenorhabditis elegans)中发现的一个miRNA基因簇的 7 个miRNA成 员集中簇生在 2 号染色体的 $800 \mathrm{bp}$ 区域内, 并且 7 个 成员之间形成旁系同源miRNA基因(图 2) ${ }^{[28]}$. 尽管很 多miRNA基因簇已经通过计算预测的方法得到确定， 但是对于这些miRNA基因簇分布的作用意义尚不是 很清楚.

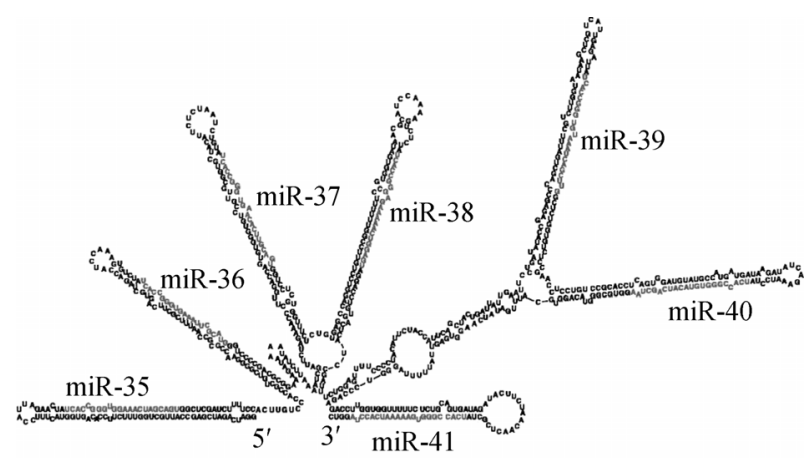

图 2 miR-35-41 家族的二级结构 28$]$

\section{2 miRNA 基因簇的表达与功能}

自从在秀丽线虫中发现两个时序性调控胚胎发 育的miRNA- - lin-4 和let-7 以来 ${ }^{[12,29]}$, 对基因表达调 控、细胞分化、发育过程调控等诸多方面涉及 miRNA 
的表达与功能的研究进入发展阶段. 在生物信息学 技术的帮助下，受miRNA调控的一系列靶基因位点 得到预测 ${ }^{[30]}$. 但目前为止，只有一小部分 miRNA的 功能作用得到较为详细的解析. 例如, 在鼠胰岛细胞 中特异性表达的 miR-375, 发挥着调控肌营养素 (myotrophin, Mtpn)基因表达的功能，并由此调控葡 萄糖介导的胰岛素胞外分泌途径 ${ }^{[31]}$; miR-208 通过调 控甲状腺激素受体相关蛋白 1(thyroid hormone receptor associated protein 1, THRAP1)的表达从而对应 激和激素信号做出响应，进而保证心脏正常的生长 发育 ${ }^{[16]}$.

从以上功能研究进展可见, 主要研究都只针对单 个miRNA的表达与功能方面，而对于miRNA基因簇的 表达与功能的研究尚处于起步阶段. 在人类和小鼠的 miRNA表达方面的研究发现，簇生排列的miRNA基因 往往构成了一个多顺反子(polycistron) $)^{[4,14,21,32]}$, 彼此 之间连同附近的蛋白编码基因一起共表达 ${ }^{[33]}$. 但是, 也有 miRNA基因簇之间的成员尽管位于同一个转录 体系中，却表现出不同的表达水平，甚至不同的表达 模式. $\mathrm{Lu}$ 等人 ${ }^{[34]}$ 对在果蝇属中发现的 miR310/311/312/313 基因簇的表达水平分析发现，除 miR-313 之外，其余 3 个miRNA都协同表达. 而对于 miR-313，发现其表达量仅为其他 3 个成员的 5\%. Yu 等人 ${ }^{[35]}$ 分析篎选得到的 51 个人类miRNA基因簇的表 达情况，发现 39 个miRNA基因簇内的miRNA成员之 间的表达相一致，剩余的 12 个miRNA基因簇内的成 员之间存在表达差异. 特别的是，对于部分处于不同 染色体上的旁系同源miRNA基因簇(paralogous clusters)却表现出了高度得表达一致性. 这种 miRNA基 因簇高度协同的表达模式，提示同源 miRNA基因簇 可能共享相同的顺式调控元件, 并发挥协同的调控 作用. 对于miRNA基因簇成员之间表达水平的差异, 其中一个可能的原因是存在着不同的转录机制或成 熟加工过程．Xu等人 ${ }^{[36]}$ 发现小鼠中 miR-212 簇、 miR-363 簇和miR-382 簇存在独立的转录机制, 并由 此产生了非一致性表达水平的结果.

既然大部分簇生排列的 miRNA 位于一个转录本 中, 那么这些 miRNA 族到底是不是比单个 miRNA 更 具有调控作用的优势呢? 从近年来的研究进展可见， 答案是肯定的.
Liu等人 ${ }^{[37]}$ 的研究提示, miR-16 家族通过调控一 系列细胞周期相关的蛋白因子, 如控制细胞周期蛋 白(Cyclin D1/D2/D3，E1/E2)和周期蛋白依赖性激酶 $(\mathrm{CDK} 2 / 4 / 6)$ 的表达，有效束缚了细胞分裂的进程. $\mathrm{Xu}$ 等人 ${ }^{[36]}$ 通过计算预测结合实验验证发现小鼠 miR-183-96-182簇通过调控胰岛素信号通路中的 3 个 因子——胰岛素受体底物 1(insulin receptor substrate 1, Irs1)、Ras p21 蛋白活化因子 1(Ras p21 protein activator 1, Rasa1)和生长因子受体结合蛋白 2(growth factor receptor bound protein 2, Grb2), 监控了整个信 号转导途径. 这种多靶点监控系统比单个 miR-375 参 与调控的葡萄糖介导的胰岛素胞外分泌途径 [31]似乎 更有效。另如在果蝇胚胎发育过程中， miR-12/ miR-283/miR-304 簇控制 Hedgehog 信号途径 ${ }^{[38]}$, miR-310s 家族和 miR-2/6/11/13/308 家族分别调控胚 胎的体轴的形成和抑制细胞调亡过程，从而保证胚 胎发育正常有序地进行 ${ }^{[39]}$. 由此可见, miRNA基因簇 承担起了调控信号网络途径的角色，这比单个 miRNA的调控模式更加复杂, 功能作用更加高效. 尽 管当前关于 miRNA基因簇生排列的原因尚未真正清 楚，但至少提示，高效精密地完成基因组表达、复制 以及协调整个相互作用网络等一系列过程，如果只 依赖逐个miRNA基因的表达来调节，往往受时间限 制. 而通过miRNA基因簇的共表达来调控整个基因 组是必要的. 同时, 由同源性的miRNA基因簇形成的 miRNA基因家族似乎更是一种午余性的自我保护 ${ }^{[35]}$, 因为一旦其中一个成员遭受突变而被淘汰, 至少还 可以确保另外几个同源的miRNA继续发挥功能. 也 许正是因为这些可能性的原因, 受选择固定后 miRNA基因簇才形成了现有的多样性分布.

\section{3 miRNA 基因簇的进化}

由于大部分的miRNA受到功能作用的制约，因 此在很大程度上, miRNA在不同物种之间是相当保守 的，这不仅反映在成熟的 miRNA序列之间高度的保 守性，还反映在形成二级结构的两翼序列的高度保 守 ${ }^{[40]}$. 正是因为 miRNA序列在不同物种之间高度的 保守性，通过不同物种间同源搜索来发现 miRNA是 一种可行的方法. Berezikov等人 ${ }^{[41]}$ 利用种系发生影像 法(Phylogenetic Shadowing)分析了灵长类物种之间 
miRNA基因的保守程度.

对于 miRNA 基因簇而言, 它们在不同物种之间 除了序列的保守性，还涉及比如基因排列的共线性 (Synteny)、重复拷贝的数量等一些进化问题. 下面将 从基因进化驱动力的角度探讨 miRNA 基因簇进化的 问题.

李昕等人 ${ }^{[42]}$ 总结了新基因的起源与进化的 4 种 基本机制——基因重复(gene duplication)、转座与逆 转座(transposition\&retrotransposition)、外显子重排 (exon shuffling)、基因水平转移(gene lateral transfer). 那么对于miRNA基因簇的进化又包括哪些机制呢? Zhang等人 ${ }^{[43]}$ 分析了位于X染色体上的miR-506 基因 族在灵长类中的进化模式，发现这个miRNA基因簇 在灵长类物种基因组中发生了串联重复的快速进化 [44]. Yu等人 ${ }^{[35]}$ 分析了 38 个miRNA基因簇内的miRNA 成员之间的同源性，发现这些簇生排列的 miRNA是 通过同一个祖先基因经过局部重复形成的. 同时分 析 38 个miRNA基因簇之间的同源性，发现 26 个基因 簇之间具有种内同源的迹象，其中有 3 个miRNA基因 家族——let-7a-1 簇、 let-7a-3 簇和 98 簇形成的种内 同源 miRNA基因家族, 29c族和 29a簇形成的家族以 及 $15 \mathrm{a}$ 簇和 16-1 簇形成的家族，每个家族内的簇之间 和簇内的成员之间依然保持着 $90 \%$ 以上的序列相似 性和染色体上排列的共线性. 由此推测, 这些miRNA 基因家族是通过片段重复而形成的. Tanzer和 Stadler ${ }^{[45]}$ 对 Mir-17 基因簇的分子进化研究, 以及 Hertel等人 ${ }^{[46]}$ 在后生动物基因组中的miRNA基因家 族之间的系统发育分析都揭示了串联重复和片段重 复是 miRNA基因簇进化的主要机制, 也因此产生了 miRNA基因簇和miRNA基因家族的多样性分布. 对 植物基因组的研究，报道了这两种重复机制也是 miRNA基因簇和基因家族进化的主要驱动力 ${ }^{[47,48]}$. 为清楚地理解重复对于 miRNA基因起源与进化的作 用，本文用简单的图示(图 3)描绘了这两种重复机制 产生miRNA基因簇的进化过程.

除了基因重复是驱动miRNA进化的主要机制, 那么其他几种进化的机制是否也可以成为 miRNA基 因簇进化的动力呢? Piriyapongsa等人 ${ }^{[49]}$ 比对分析了 462 个人类miRNA基因与转座元件之间的相似性，结 果发现有 55 个miRNA基因是由转座而产生的.
Zhang等人 ${ }^{[50]}$ 分析了位于 19 号染色体上的灵长类特 异性的miRNA基因家族，发现该家族在灵长类物种 内的排列差异是由Alu转座元件所造成的, 并由此产 生了物种特异性的miRNA获得与丢失(gain and loss) 的分子进化过程. 在果蝇属中的miRNA基因的进化 研究也揭示了 miRNA基因在物种内特异性的产生与 消亡的进化过程 ${ }^{[34,51]}$. 由此说明, miRNA基因簇起源 与进化的过程也可以是由转座或者逆转座介导的.

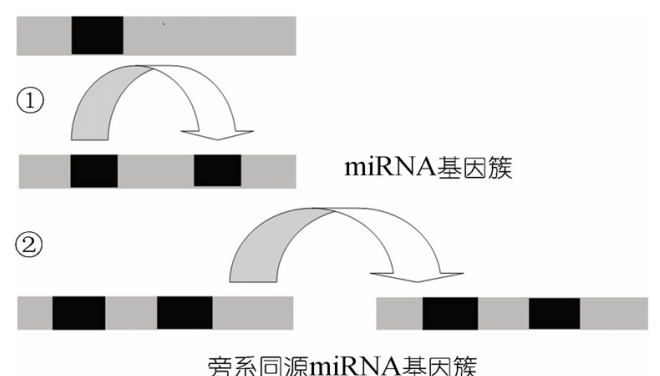

图 3 miRNA 基因簇的进化过程

(1) 表示串联重复; (2) 表示片段重复. 黑色方框代表 miRNA 基因

众所周知，线粒体基因组中的基因可以通过水 平转移而嵌入核基因组中. miRNA基因是否可以通过 这种方式产生呢? 有研究 ${ }^{[52]}$ 分析了 13 个真核生物基 因组中存在的线粒体假基因 (nuclear mitochondrial DNA, NUMTs)与非编码DNA之间的可能性关系，结 果显示，两者之间并没有严格的相关性. 该结果表明, 通过基因组之间的水平基因转移途径产生miRNA基 因的可能性是比较小的. 因为水平基因转移整合到 宿主基因组内并参与宿主基因组表达调控的过程是 受到强烈的自然选择影响的 ${ }^{[53]}$.

综上所述，尽管对于 miRNA 基因成簇排列的功 能意义目前尚未完全清楚，但通过对其进化机制的 分析和部分 miRNA 基因簇的表达与功能作用的研究 提示, miRNA 基因簇的形成不是随机性的，而是在自 然选择过程中逐渐积累并得到固定的结果. 本文将 通过具体的实例来进一步说明呈簇生排列的 miRNA 基因在选择过程中产生与消亡的适应性更替，以及 这种物种内特异性的进化历程对于物种演变的指导 性意义.

\section{4 实例分析}

达尔文正选择是加速基因进化并在此基础之上 
产生新基因的主要选择方式. miRNA基因的起源与进 化问题也随着大量miRNA的发现而受到关注. 有假 设认为，与靶基因之间的协同作用是miRNA基因从 头衍生(de novo generation)和进化的主要来源 ${ }^{[47,54]}$. $\mathrm{Lu}$ 等人 ${ }^{[34]}$ 分析了果蝇属 12 个物种之间的miRNA基因 的起源与进化过程, 发现miRNA基因在这 12 个物种 内，至少演化出了 5 个miRNA新基因，其中 4 个 Mir-310/311/312/313 成簇排列构成了一个基因簇. 通 过推算分歧时间，发现该基因簇是在果蝇亚属 (Subgenus Drosophila)和水果果蝇亚属(Subgenus Sophophora) 分歧的过程中起源的. 另外一个Mir-303 发生在黑腹果蝇亚组形成的过程中. 已有文献 ${ }^{[39]}$ 报 道, Mir-310 基因簇在胚胎发育的体轴形成过程中扮 演着关键的作用。这似乎表明，果蝇亚属内Mir-310 基因簇的起源可能就是导致种属分化的一个原因. 大规模小RNA的测序分析同样表明，miRNA基因在 果蝇属内发生了多次从头起源与进化的过程，并且 表现出物种特异性的产生与消亡的进化过程. 尤其 在拟果蝇(Drosophila simulans)物种形成过程中, 发 现平均每百万年约有 12 个miRNA基因起源，经过选 择压力的清除, 最终净产生 0.3 个miRNA基因 ${ }^{[51]}$. 由 此可知，在物种形成过程中，除了控制表型特征的蛋
白质编码基因发挥了关键的作用外，这些在基因组 表达水平上发挥调控作用的 miRNA 基因, 对于物种 形成的作用同样重要.

对灵长类物种的研究也表明, miRNA基因簇受 到了加速进化同时伴有种系内重复扩增和丢失的适 应性变化过程 ${ }^{[43,50]}$. 进一步印证了 miRNA基因簇通 过调控基因组表达网络的模式推动着物种的演变.

\section{5 展望}

miRNA 基因的协同作用可能是 miRNA 基因簇 演化的一种动力. 作为基因组中的 “暗物质”, miRNA 基因簇如此复杂的调控方式是如何在 miRNA 基因的 产生与消亡的更替中演变的，以及为什么 miRNA 基 因簇会发生这种更替的适应性变化和它们的功能意 义如何, 都是解析生命体复杂性的奥秘所在. 因此, 为理解 miRNA 基因簇的网络调控模式与它们自身进 化的关系, 需要凭借系统发育基因组学的方法, 来阐 明某一种系物种基因组中 miRNA 基因簇的产生(或 消亡)是否获得(或丢失)了一些调控靶点, 这些调控 靶点在生命机体内扮演了什么角色，在遗传相互作 用网络中又发挥了什么功能. 进一步说, 对 miRNA 网络调控模式的研究将推动以 miRNA 基因簇为依托 的药物开发和解析复杂性疾病的发生机制.

\section{参考文献}

1 Dennis C. The brave new world of RNA. Nature, 2002, 418(6894): 122-124[DOI]

2 Lee Y, Lee Y, Kim M, et al. MicroRNA genes are transcribed by RNA Polymerase II . EMBO J, 2004, 23(20): 4051-4060[DOI]

3 Borchert G M, Lanier W, Davidson1 B L. RNA polymerase Ill transcribes human microRNAs. Nat Struct Mol Biol, 2006, 13(12): $1097-1101[\mathrm{DOI}]$

4 Bartel D P. MicroRNAs: genomics, biogenesis, mechanism, and function. Cell, 2004, 116(2): 281 -297 [DOI]

5 Han J, Lee1 Y, Yeom K H, et al. Molecular basis for the recognition of primary microRNAs by the Drosha-DGCR8 complex. Cell, 2006, 125(5): 887-901[DOI]

6 Bohnsack M T, Czaplinski K, Gorlich D. Exportin 5 is a RanGTP-dependent dsRNA-binding protein that mediates nuclear export of pre-miRNAs. RNA, 2004, 10(2): 185-191 [DOI]

7 Brennecke J, Stark A, Russell R B, et al. Principles of microRNA-target recognition. PLoS Biol, 2005, 3(3): e85[DOI]

8 Lai E C. Micro RNAs are complementary to 3'UTR sequence motifs that mediate negative post-transcriptional regulation. Nat Genet, 2002, 30(4): 363-364[DOI]

9 Selbach M, Schwanhausser B, Thierfelder N, et al. Widespread changes in protein synthesis induced by microRNAs. Nature, 2008, 455(7209): 58-63[DOI]

10 Krek A, Grun D, Poy M N, et al. Combinatorial microRNA target predictions. Nat Genet, 2005, 37(5): 495-500[DOI]

11 Reinhart B J, Slack F J, Basson M, et al. The 21-nucleotide let-7 RNA regulates developmental timing in Caenorhabditis elegans. Nature, 2000, 403(6772): 901-906 [DOI]

12 Lee R C, Feinbaum R L, Ambros V. The C.elegans heterochronic gene lin-4 encodes small RNAs with antisense complementarity to 
lin-14. Cell, 1993, 75(5): 843-854 $\underline{\text { [DOI] }}$

13 Felli N, Fontana L, Pelosi E, et al. MicroRNAs 221 and 222 inhibit normal erythropoiesis and erythroleukemic cell growth via kit receptor down-modulation. Proc Natl Acad Sci USA, 2005, 102(50): 18081-18086[DOI]

14 Ambros V. The functions of animal microRNAs. Nature, 2004, 431(7006): 350-355[DOI]

15 Huang J L, Wang F X, Argyris E, et al. Cellular microRNAs contribute to HIV-1 latency in resting primary CD4 ${ }^{+} \mathrm{T}$ lymphocytes. Nat Med, 2007, 13(10): 1241-1247 [DOI]

16 Van R E, Sutherland L B, Qi X, et al. Control of stress-dependent cardiac growth and gene expression by a microRNA. Science, 2007, 316(5824): 575-579 [DOI]

17 Esquela-Kerscher A, Slack F J. Oncomirs-microRNAs with a role in cancer. Nat Rev Cancer, 2006, 6(4): 259-269[DOI]

18 Chang T C, Mendell J T. microRNAs in vertebrate physiology and human disease. Annu Rev Genom Human Genet, 2007, 8: 215$239 \underline{\text { [DOI] }}$

19 Hébert S S, De Strooper B. miRNAs in neurodegeneration. Science, 2007, 31(317): 1179—1180 [DOI]

20 Yang B, Lin H, Xiao J, et al. The muscle-specific microRNA miR-1 regulates cardiac arrhythmogenic potential by targeting GJA1 and KCNJ2. Nat Med, 2007, 13(4): 486-491[DOI]

21 Lagos-Quintana M, Rauhut R, Meyer J, et al. New microRNAs from mouse and human. RNA, 2003, 9(2): 175-179 [DOI]

22 Lai E C, Tomancak P, Williams R W, et al. Computational identification of Drosophila microRNA genes. Genome Biol, 2003, 4(7): R42[DOI]

23 Lewin B. Genes VIII. New Jersey: Pearson Education Inc., 2004

24 Altuvia Y, Landgraf P, Lithwick G, et al. Clustering and conservation patterns of human microRNAs. Nucleic Acids Res, 2005, 33(8): $2697-2706 \underline{[\mathrm{DOI}]}$

25 Megraw M, Sethupathy P, Corda B, et al. miRGen: a database for the study of animal microRNA genomic organization and function. Nucleic Acids Res, 2007, 35: D149-155[이]

26 Tang G Q, Maxwell E S. Xenopus microRNA genes are predominantly located within introns and are differentially expressed in adult frog tissues via post-transcriptional regulation. Genome Res, 2008, 18: 104-112[DOI]

27 Mendell J T. miRiad roles for the miR-17-92 cluster in development and disease. Cell, 2008, 133(2): 217-222[DOI]

28 Lau N, Lim L, Weinstein E, et al. An abundant class of tiny RNAs with probable regulatory roles in Caenorhabditis elegans. Science, 2001, 294(5543): 858-862[DOI]

29 Pasquinelli A, Reinhart B, Slack F, et al. Conservation of the sequence and temporal expression of let-7 heterochronic regulatory RNA. Nature, 2000, 408(6808): 86-89 [DOI]

30 John B, Enright A J, Aravin A, et al. Human microRNA targets. PLoS Biol, 2004, 2(11): e363[DOI]

31 Poy M N, Eliasson L, Krutzfeldt J, et al. A pancreatic islet-specific microRNA regulates insulin secretion. Nature, 2004, 432(7014): $226-230[\mathrm{DO}]$

32 Cullen B R. Transcription and processing of human microRNA precursors. Mol Cell, 2004, 16(6): 861-865[DOI]

33 Baskerville S, Bartel D P. Microarray profiling of microRNAs reveals frequent coexpression with neighboring miRNAs and host genes. RNA, 2005, 11(3): 241-247 [DOI]

34 Lu J, Fu Y, Kumar S, et al. Adaptive evolution of newly emerged microRNA genes in Drosophila. Mol Biol Evol, 2008, 25(5): 929$938 \underline{[\mathrm{DOI}]}$

35 Yu J, Wang F, Yang G H, et al. Human microRNA clusters: genomic organization and expression profile in leukemia cell lines. Biochem Biophys Res Commun, 2006, 349(1): 59-68[DOI]

$36 \mathrm{Xu} \mathrm{J} \mathrm{Z,} \mathrm{I} \mathrm{Wong} \mathrm{C} \mathrm{W.} \mathrm{A} \mathrm{computational} \mathrm{screen} \mathrm{for} \mathrm{mouse} \mathrm{signaling} \mathrm{pathways} \mathrm{targeted} \mathrm{by} \mathrm{microRNA} \mathrm{clusters.} \mathrm{RNA,} \mathrm{2008,} \mathrm{14(7):} \mathrm{1276-}$ $1283 \underline{\text { DOI] }}$

37 Liu Q, Fu H, Sun F, et al. miR-16 family induces cell cycle arrest by regulating multiple cell cycle genes. Nucleic Acids Res, 2008, 36(16): 5391-5404 [DOI]

38 Friggi-Grelin F, Lavenant-Staccini L, Therond P. Control of antagonistic components of the hedgehog signaling pathway by microRNAs in Drosophila. Genetics, 2008, 179(1): 429-439 [DOI]

39 Leaman D, Chen P Y, Fak J, et al. Antisense-mediated depletion reveals essential and specific functions of microRNAs in Drosophila development. Cell, 2005, 121(7): 1097-1108[DOI]

40 Ohler U, Yekta S, Lim L P, et al. Patterns of flanking sequence conservation and a characteristic upstream motif for microRNA gene 
identification. RNA, 2004, 10(9): 1309-1322 [DOI]

41 Berezikov E, Guryev V, van de Belt J, et al. Phylogenetic shadowing and computational identification of human microRNA genes. Cell, 2005, 120(1): 21-24[DOI]

42 李昕, 杨爽, 彭立新, 等. 新基因的起源与进化. 科学通报, 2004, 49(13): 1219-1225

43 Zhang R, Peng Y, Wang W, et al. Rapid evolution of an X-linked microRNA cluster in primates. Genome Res, 2007, 17(5): 612$617 \underline{\text { [DOI] }}$

44 Su B, Zhang R. MicroRNA evolution in the human genome. Encyclopedia of Life Sciences, 2008. doi: 10.1002/9780470015902. a0020788

45 Tanzer A, Stadler P F. Molecular evolution of a microRNA cluster. Mol Biol, 2004, 339(2): 327-335[DOI]

46 Hertel J, Lindemeyer M, Missal K, et al. The expansion of the metazoan microRNA repertoire. BMC Genomics, 2006, 7: 25[DOI]

47 Li A, Mao L. Evolution of plant microRNA gene families. Cell Research, 2006, 17: 212-218

48 Maher C, Stein L, Ware D. Evolution of arabidopsis microRNA families through duplication events. Genome Res, 2006, 16(4): 510519 [DOI]

49 Piriyapongsa J, Marino-Ramirez L, Jordan I K. Origin and evolution of human microRNAs from transposable elements. Genetics, 2007, 176(2): 1323-1337 [DOI]

50 Zhang R, Wang Y Q, Su B. Molecular evolution of a primate-specific microRNA family. Mol Biol Evol, 2008, 25(7): 14931502[DOI]

51 Lu J, Shen Y, Wu Q, et al. The birth and death of microRNA genes in Drosophila. Nat Genet, 2008, 40(3): 351-355[DOI]

52 Richly E, Leister D. NUMTs in sequenced eukaryotic genomes. Mol Biol Evol, 2004, 21(10): 1081—1084 [DOI]

53 Lercher M J, Pál C. Integration of horizontally transferred genes into regulatory interaction networks takes many million years. Mol Biol Evol, 2008, 25(3): 559-567 [DOD]

54 Bartel D P, Chen C Z. Micromanagers of gene expression: the potentially widespread influence of metazoan microRNAs. Nat Rev Genet, 2004, 5(5): 396-400[이] 